OPEN ACCESS

Citation: Cingolani P. (2021) L'informalità del capitale: tempi, lavoro e capitalismo delle piattaforme, in "Cambio. Rivista sulle trasformazioni sociali», Vol. 11, n. 21: 79-89. doi: 10.36253/cambio-10903

Copyright: (c) 2021 Cingolani P. This is an open access, peer-reviewed article published by Firenze University Press (http://www.fupress.com/cambio) and distributed under the terms of the Creative Commons Attribution License, which permits unrestricted use, distribution, and reproduction in any medium, provided the original author and source are credited.

Data Availability Statement: All relevant data are within the paper and its Supporting Information files.

Competing Interests: The Author(s) declare(s) no conflict of interest.
Monographic Section

\section{L'informalità del capitale: tempi, lavoro e capitalismo delle piattaforme}

\author{
Patrick Cingolani \\ Université de Paris \\ cingolanipatrick@gmail.com \\ (traduzione a cura di Domenica Farinella)
}

\begin{abstract}
The outsourcing movement of the second half of the 20th century had fueled informality on its edges: illegal workers in European construction companies or sweatshops of subcontractors from developing countries with unsanitary buildings and degraded working conditions. Digitization in the 21 st century has reconfigured this movement in an original way, shifting work towards the home or towards the sphere of everyday life. The article attempts to explain the ways in which capitalism invades spheres and areas that sometimes remained relatively unspoiled or free: random mobility of the driver or the rider of the platforms; interstitial temporality of the "turkers" or "click workers", space for staging oneself or for gaming on YouTube; amateur management at Lego. The informality of capital is here the effect of the original process of market colonization of the interstitial time in everyday life.
\end{abstract}

Keywords: colonization of everyday life, outsourcing, piecework, platforms, spatial and temporal labour boundaries, gigs.

Dalla fine degli anni Ottanta, in seguito alle riforme neoliberali, l'impresa tende a deterritorializzarsi e, con le successive spinte verso la digitalizzazione, a dematerializzarsi (Weil 2014; Davis 2016). In questo articolo una minore enfasi sarà data agli aspetti finanziari e giuridici di questa dematerializzazione, per concentrarci sugli effetti in ambito organizzativo. In particolare, il processo di outsourcing non solo deterritorializza l'azienda segmentandola, ma sposta anche i suoi dispositivi di controllo e colonizzazione verso la sfera del domestico e del quotidiano. Il telelavoro o, più in generale, il lavoro a distanza non sono che alcuni esempi di un processo che si appropria di attività e ambiti finora rimasti fuori dal dominio del mercato: il gioco e, più in generale, le manifestazioni amatoriali e l'hobbistica nei settori più disparati, dall'artigianato tradizionale ai blog e ai post sulle piattaforme online (videomaker di moda o reporter di notizie su YouTube, per esempio). 
La digitalizzazione e l'intermediazione di piattaforme stanno estendendo lo spettro della mercificazione, aprendole spazi che fino ad ora erano relativamente "vergini", perché riguardavano attività e legami domestici o vernacolari ${ }^{1}$. Esse si impongono su queste stesse sfere attraverso forme di controllo e di sorveglianza che permettono lo sfruttamento di una forza lavoro delocalizzata e globalizzata (Amazon Mechanical Turk, $U_{p}$ Work) o di una forza lavoro locale, disponibile a richiesta (Helping, My Hammer, etc.). Questi diversi aspetti costituiscono quella che noi chiamiamo l'informalità del capitale. Si tratta, attraverso questa espressione, di pensare la categoria di informalità in relazione alla singolare possibilità apportata al capitale dalle nuove tecnologie di comunicazione (NITC) di infiltrare forme non-organizzative ed aleatorie di cooperazione produttiva e di socializzazione, la cui conseguenza è appunto colonizzare le sfere domestiche (Anderson 2008; Shirky 2009). Due esempi paradigmatici di questa informalità saranno sviluppati lungo l'articolo.

\section{SPOSTARE LA FRONTIERA, CONQUISTARE I TERRITORI}

1) L'informalizzazione dei confini. Grazie alla loro capacità pervasive le NTIC hanno sovvertito i confini e i limiti che erano stati al centro della morfologia sociale del fordismo (Cingolani 2016). L'organizzazione sociale del $\mathrm{XX}$ secolo è stata caratterizzata dalla separazione tra lo spazio professionale e quello domestico - separazione in cui vi era sia una protezione dello spazio privato di fronte alle intrusioni del lavoro, che una difesa della sfera dell'intimità dal mercato. Durante il XX secolo la commercializzazione delle produzioni domestiche restava marginale nelle grandi metropoli occidentali e le attività cooperative operaie sottendevano spesso degli scambi non di mercato (Weber 1985); al contrario, nel XXI secolo, la digitalizzazione banalizza e individualizza la monetizzazione dei tempi di attività interstiziali. Noi ci interesseremo al superamento dei limiti tra lavoro e casa, così come alla sovversione dei segni e dei marcatori storici di questi limiti. Al di là della sola sfera domestica, vi è la sfera del quotidiano che è caratterizzata da una moltitudine di micro-task che vengono proposti dalle piattaforme (Casilli 2019). Ma, in una prospettiva più ampia, sarebbe possibile considerare il saccheggio a fini di commercializzazione della sfera privata realizzato dalle piattaforme di data mining e dalle tecnologie che invadono sempre più l'intimo e le attività quotidiane, siano esse attività all'aperto (jogging, spostamenti, ecc.) o a casa (tra gli altri, attraverso le nuove applicazioni domotiche) (Zuboff 2020). Di fatto, noi intendiamo informale e informalità in una opposizione dialettica con un quadro economico e sociale regolamentato dall'azione legislative e dall'intervento dello Stato (Sassen 1997). La riconfigurazione provocata dalle piattaforme apre degli spazi, dei tempi, delle attività, a volte infinitesimali, rilevanti un infra-diritto e dei vuoti regolamentari. Aprendo nuove frontiere e nuovi bacini da cui attingere tempo e informazioni, le piattaforme producono delle zone che sono di fatto senza diritti. Anche se la maggior parte dei click work sono effettuati nei paesi del Sud (soprattutto l'India o le Filippine) (ILO 2021), l'introduzione della piattaforma associata all'autoimprenditorialità e al lavoro autonomo minano progressivamente il modello di un lavoratore protetto nei paesi del Nord, riconfigurando la struttura temporale del lavoro. Le figure emergenti del lavoratore a distanza si sono diffuse significativamente negli Stati Uniti, in Canada, nel Regno Unito, in Ucraina, in Russia. Esse si associano alla effettiva crescita in tutta Europa e nel mondo di numerose piattaforme che riguardano attività non delocalizzabili (NCC - noleggio con conducente, consegna e facchinaggio, artigianato, lavori domestici) per provocare degli effetti distruttivi sulle regolazioni sociali.

2) La colonizzazione del gratuito e del quotidiano ${ }^{2}$. In parallelo alle nuove porosità dei confini che nel passato recente hanno costituito la realtà morfologica del lavoro salariato, nuovi spazi sono oggetto di mercificazione e

\footnotetext{
${ }^{1}$ In questo articolo, il termine vernacolare è inteso sia nella sua relazione etimologica con ciò che si fa in casa, sia nella sua dimensione d'uso proprio, non di mercato. Un significato ripreso in particolare da Ivan Illich nella sua opera (Illich 2005 e Paquot 2012), ma che trova anche eco in Michel de Certeau e nel suo pensiero su tattiche e «bracconaggio» (Certeau 1980).

${ }^{2}$ Gli avanzamenti teorici su questo tema sono stati possibili grazie alle riflessioni che da Henri Lefebvre fino a Guy Debord, passando per De Certeau, hanno studiato la vita quotidiana e il suo funzionamento in modo critico. Più precisamente è Guy Debord che evoca la colonizzazione del quotidiano nell' internazionale situazionista (Debord 1961). Si troverà una eco delle sfide lanciate da queste analisi nel mio recente libro sul capitalismo di piattaforma (Cingolani 2021).
} 
sfruttamento fino a una colonizzazione. Da un lato (2.1), nuove tensioni si producono nei rapporti tra lavoro e capitale, rendendo possibili diverse forme di utilizzazione (o meglio di cooptazione) di attività gratuite, che si realizzano sia attraverso un'economia della promessa e del "miraggio di assunzione", sia tramite l'appropriazione di attività espressamente gratuite. Gli stage, il volontariato e altre attività più recenti, come il woofing, in cui le persone vanno a lavorare gratuitamente presso gli agricoltori, sono sempre più in tensione tra prestazione gratuita e logiche di mercato (Simonet 2018). Certi settori sono più particolarmente caratterizzati dallo sfruttamento del lavoro gratuito, ad esempio quelli associati all'arte e alle industrie culturali o la moda. Alla flessibilità e a contratti brevi e precari si affianca il lavoro gratuito, offerto da chi vuole entrare in mercati molto chiusi e crearsi un'immagine in un mondo largamente fondato sulla reputazione. Per la costruzione del curriculum nell'ambito di una carriera professionale, per fare una prima esperienza di lavoro, bisogna pagare il prezzo di un comportamento oblativo e mostrare un certo servilismo (Ross 2009). In modo evidente, se l'appropriazione delle attività "gratuite" si scontra con limiti giuridici e normativi, è piuttosto l'indebolimento della sua eterogeneità rispetto all'attività economica e il rafforzamento della sua dipendenza dal mercato che offrono delle occasioni di assorbimento di una ricchezza prodotta gratuitamente; così come è anche il fluttuare sempre più ampio dei marcatori di differenze e di separazione tra tempo libero e di lavoro che conduce a una larga sussunzione dei tempi sociali all'accountability (2.2). Internet come un nuovo spazio di investimento ha innanzitutto aperto degli spazi di attività libera, associati alla comunicazione e all'espressività (blog, giochi, messa in scena di sé, attività relazionali, ecc), estendendosi in una certa misura in un ambito ancora "vergine", informale e libero da vincoli giuridici. Tuttavia, questo spazio è diventato sempre più l'oggetto di relazioni mercificate e di contratti di mercato che ricompongono i rapporti di lavoro e i loro statuti professionali, come mostrano le nuove forme di lavoro indipendente o autonomo e di microimprenditorialità digitale. I pay per click (ovvero i pagamenti legati al numero di visualizzazioni o di like), la professionalizzazione e la remunerazione degli youtuber da parte delle piattaforme a partire dai loro guadagni pubblicitari, i loro incoraggiamenti a portare con sé, esporre, raccomandare, consumare dei prodotti on line sono altrettante espressioni di un rapporto nuovo tra capitalismo e informalità. La proprietà dei supporti digitali da parte delle piattaforme informatiche permette poi nuove forme di abuso dell'attività creativa degli utenti-fruitori. Sembra configurarsi uno spazio misto, ibrido, di attività e di lavoro e di leisure, di mercato e non mercato, di permeabilità costante tra gratuito e remunerato.

\section{LA DETERRITORIALIZZAZIONE DELL'IMPRESA}

Il movimento di ristrutturazione e riconfigurazione dell'impresa che è iniziato attorno agli anni SettantaOttanta ha rivoluzionato le caratteristiche morfologiche del fordismo, a cominciare dai confini che delimitavano lo spazio del lavoro e quello del non-lavoro, sovvertendo il quadro normativo che si era progressivamente affermato nel ventesimo secolo. L'età del capitalismo burocratico di cui M. Weber aveva anticipato i tratti negli anni Venti si era cristallizzata attorno alla fabbrica come un luogo chiuso, con la sua unità di tempi e luoghi di lavoro. Verso la fine del ventesimo secolo, la disintegrazione verticale e l'esternalizzazione delle imprese riconfigurano l'esperienza concreta dei confini del lavoro. L'offshoring e il subappalto trasformano la territorialità dell'impresa e separano la sua unità di luogo, aprendola verso i paesi in via di sviluppo e verso condizioni opache di lavoro (che talvolta rientrano nelle norme infra-giuridiche in materia di occupazione o di sicurezza), oppure frammentandola, delegando a delle piccole imprese locali i ritmi e i vincoli della produzione a condizioni lavorative e salariali minime (Weil 2014). La flessibilizzazione dell'organizzazione del lavoro infrange l'esperienza della temporalità dell'impresa e ne opacizza le condizioni di lavoro e d'impiego (Cingolani 2017). I lavoratori occasionali, gli intermittenti (interinali, i contratti a tempo determinato, i lavoratori part-time) vanno ad occupare gli interstizi, le lacune del processo lavorativo, mentre gli accordi tra interinale e subappalto consentono di contravvenire ai diritti del lavoro, creando delle "maschere" giuridiche e occultando le responsabilità delle imprese - ad esempio permettendo l'assunzione a giornata (o anche ad ore) lavoratori irregolari (Jounin 2008).

Ormai il movimento di esternalizzazione tende a sfociare in una colonizzazione che di fatto è un cambiamento fondamentale nell'evoluzione del capitale. Le logiche di subappalto di ieri sono completate dall'utilizzo dei dispositi- 
vi wireless di individualizzazione del lavoro di cui le figure del lavoratore indipendente o del microimprenditore sono le forme giuridiche. Anche se i lavoratori possono utilizzare in diversi modi questi dispositivi, essi sono anche la condizione per il saccheggio della loro sfera di libertà. Ma già certe forme di lavoro a domanda come il "lavoro a chiamata", il "contratto a zero ore" in Inghilterra o il lavoro a distanza hanno colonizzato il tempo domestico e privato. L'attesa incerta di un impiego di qualche ora, che può essere interrotto all'ultimo minuto da un semplice SMS implica una messa a disposizione del tempo di non lavoro. Quei confini che il secolo precedente, in risposta alle lotte operaie, aveva distinto in modo molto più netto sono sempre più porosi e il capitalismo appare sempre più alla ricerca di tutte le temporalità informali liberate dalla riduzione dell'orario di lavoro formale socialmente distribuito.

\section{IL CAPITALISMO DI PIATTAFORMA: DALLA FRAMMENTAZIONE ALLA TASKIFICAZIONE DEL LAVORO}

In continuità con i dispositivi di flessibilizzazione e precarizzazione del XX secolo, la piattaforma opera come un mediatore tra lavoratore e committente senza la funzione istituzionale di integrazione tra le parti. Nel trasformare, il lavoratore e il cliente in "utenti", da un lato la piattaforma nega il rapporto sociale di subordinazione e il quadro giuridico e istituzionale ad esso associato, dall'altro apre nuove possibilità di messa in rete tra "utenti" in sfere d'attività fino ad allora non oggetto di mercificazione (Casilli 2019). La possibilità di monitorare la manodopera attraverso l'algoritmo, senza una strutturazione organica, porta ad un'infinità di compiti che non potrebbero essere svolti in un'impresa, all'interno di un rapporto contrattuale tradizionale o dai quali non si sarebbe potuto ricavare alcun profitto. Camminare in strada con una videocamera (Google view), fotografare dei beni di consumo sulle mensole del supermarket (Click and Walk), rispondere a indagini online, identificare certi articoli su un insieme disparato di beni di consumo (Amazon Mechaincal Turk) diventano da questo momento delle attività remunerabili e realizzabili per conto di un cliente. A crollare non è tanto il sistema salariale, quanto tutta la temporalità che lo aveva consentito e che aveva fatto da contraltare alla sfera vernacolare fuori mercato. Dobbiamo qui ricordare la celebrazione della domenica da parte di Proudhon (Proudhon 1839) e il ruolo storico del movimento operaio per la riduzione del tempo di assoggettamento alle logiche di mercato. La possibilità di mobilitare questa sfera storicamente non mercificata a fini di profitto diventa l'occasione per un'invasione opportunista della vita quotidiana.

Il processo avviato da Uber o Deliveroo attraverso l'intermediazione di una forza lavoro di autisti o fattorini sembra essere la parte più visibile dell'informalizzazione del capitale.

A partire dalla governance algoritmica, il principio di Uber pop si basava sulla mobilitazione di chiunque. Anche se proibito per alcuni anni dalla Corte di giustizia europea, il suo inizio mostra con chiarezza l'effetto di informalizzazione delle piattaforme. Se oggi il tassista di Uber è un conducente iscritto in un registro amministrativo, titolare di un'assicurazione professionale, nel quadro di Uber pop, chiunque poteva, attraverso una semplice applicazione sullo smartphone, improvvisarsi tassista e trasportare passeggeri. Ancora oggi la durata del lavoro non è regolamentata. Il meccanismo del surge pricing può sollecitare l'autista in qualsiasi momento in un contesto di intensificazione dell'attività: i prezzi visualizzati sulla sua app durante le ore di punta lo incoraggiano a continuare a lavorare (Rosenblat 2018).

Ad esempio, ancora oggi in Francia, chiunque può diventare rider per Deliveroo o fare il corriere per qualche altra piattaforma di consegna, inscrivendosi al registro dei lavoratori autonomi. L'orario di lavoro è a discrezione del lavoratore, ma dietro questo spazio apparentemente libero e aperto che è la strada, la piattaforma ne assicura la gestione algoritmica: decidendo le consegne, regolando e monitorando i tempi, monitorando il corriere con il GPS. Allo stesso modo, le piattaforme di gig work (YoupiJob, Needhelp, Taskrabbit, ecc.) rendono mercificabili una serie di compiti che, senza l'intermediazione di una piattaforma, non sarebbero stati redditizi per dei professionisti.

Questi compiti non sono particolarmente interessanti per artigiani e professionisti perché implicano servizi o attività talmente piccoli da non essere "redditizi" e per questo vengono lasciate a principianti o a lavoratori amatoriali: Amazon Mechanical Turk (AMT), Figure Eight negli Stati Uniti, Foule Factory in Francia, offrono micro-task a volte sequenziati fino a un'estrema frammentazione che possono essere eseguite «alla fermata dell'autobus o in una sala d'attesa» (Barraud de Lagerie e Sigalo Santos 2018). 
Trascrivere scontrini accartocciati, tradurre una scheda prodotto dall'inglese all'arabo, identificare delle persone in cinque foto, verificare che gli articoli in vendita su un sito di e-commerce abbiano le giuste parole chiave o buone immagini, sono tutte attività provenienti da piattaforme di micro-work come Amazon Mechanical Turk che un turker può svolgere a qualsiasi ora del giorno o della notte, a casa o in un internet point. Il processo di informalizzazione comporta la possibilità di colonizzare spazi non di mercato con merci, che facevano parte di un'economia vernacolare (fai-da-te) o che fino ad allora non avevano valore prettamente economico perché non organizzabili. Si realizza così un' informalizzazione del capitale perché quest'ultimo utilizza la piattaforma come uno strumento di sovversione del rapporto contrattuale caratteristico del lavoro salariale, fino ad arrivare all'effetto più radicale di svuotare di senso e significato ciò che prima veniva identificato come lavoro o come relativo ad un impiego.

\section{PREDAZIONE E PARASSITISMO DEL GRATUITO}

Questo allargamento della sfera del lavoro su quella privata, nelle temporalità quotidiane finora non mercificate, rappresenta in effetti il sovvertimento capitalistico della categoria di lavoro. Se il lavoro è dappertutto, non è da nessuna parte, perché l'incertezza sulla sua natura, l'erosione dei suoi criteri di definizione lo rendono non più identificabile come tale, alimentando così tutte le possibili forme capitalistiche di sovversione del rapporto salariale e, più in generale, del "rapporto di lavoro", in cui il sentimento di eteronomia o addirittura di alienazione è stato per lungo tempo il criterio.

Il lavoro non si trova praticamente da nessuna parte perché il capitalismo delle piattaforme ha cercato di minare la relazione sociale del lavoro. La piattaforma opera come un intermediario tra utenti e quindi non impiega nessuno. Ad esempio, i difensori di Uber continuano a dire «facciamo soldi con le licenze software». Uber non è un'azienda di trasporti, si presenta come un'azienda tecnologica che affitta l'utilizzo del proprio software agli autisti (Rosenblat 2018). Come attestano gli scambi tra le varie piattaforme e gli autisti o i corrieri, le parole associate a un rapporto di lavoro (come «assumere., «impiegare» e tutte le espressioni che potrebbero ricordare un rapporto di subordinazione) sono costantemente negate. La rappresentazione immaginaria dell'attività deve essere quella della libertà, della scelta, dell'autonomia, e l'idea di partnership sostituisce un contratto che esprime asimmetria. Inoltre, le piattaforme rendono incerta la categoria stessa di lavoro e i segni linguistici di un rapporto di subordinazione, come il rendimento a quale si preferisce lo score, o ancora i riferimenti a una gerarchia scompaiono di fronte a sollecitazioni automatiche. Ovviamente dal rapporto di subordinazione si procede alla gamification del lavoro, ma anche alla negazione della sua dimensione eteronomica e noiosa. Si tratta quindi di messaggi di sfida generati automaticamente da un algoritmo che consentono di evitare ingiunzioni e ordini che sono propri del comune regime di subordinazione lavorativa. Si incitano i tassisti a guadagnare di più, piegandosi alle sfide offerte dall'algoritmo come: «Fai 34 viaggi tra le 5 del mattino di domenica e le 5 di lunedì per ricevere un bonus di 63 dollari» (O'Connor 2013). In questo senso, i giochi, i loro punteggi, offrono un'esperienza istantanea e viscerale di successo e ricompensa, mobilitando la dimensione emotiva e istintiva del lavoratore. Se l'introduzione del gioco nel lavoro non è nuova nella storia del management, l'originalità qui sta nel fatto che tale discorso è insito nell'ideologia stessa della piattaforma come negazione del rapporto di lavoro e di subordinazione. Il senso di cosa significa lavoro viene opportunamente evitato, mentre il discorso della sharing economy assume i connotati di un'apparente "cooperazione" e socializzazione tra utenti: «Uber è un nuovo modo popolare per guadagnare soldi extra dando alle persone passaggi con la propria auto». Questa crisi della rappresentazione storica del lavoro è di rara forza perché combina la base materiale di una macchina algoritmica astratta con l'artificio ideologico della condivisione tra utenti (Arcidiacono et al., 2018). Non è tanto importante sapere se i lavoratori di piattaforma siano o no ingannati da questa retorica, ma sottolineare la forza di questo capitalismo e il suo potere penetrante sulla quotidianità, che agisce naturalizzando e banalizzando il processo di sovvertimento dei segni (e quindi la fine della distinzione tra lavoro e non lavoro) ${ }^{3}$.

\footnotetext{
${ }^{3}$ In California, le piattaforme VTC, insieme alle piattaforme di consegna, hanno speso 200 milioni di dollari per evitare la possibilità che i loro "utenti" fossero riqualificati come dipendenti e alla fine hanno vinto la causa. (Kesselmann, 2020).
} 
Nel movimento congiunto della piattaforma e della sua capacità di minare confini e significati, vi è la possibilità di trasformare qualsiasi attività in lavoro o comunque in una fonte di estrazione di valore, ad esempio, come vedremo nel proseguo dell'articolo, quando si crea un profitto sfruttando attività online che erano nate come gratuite e condivise, o ancora nell'immanenza di un rapporto di confusione tra gioco, creatività e mercato. Se il lavoro non è da nessuna parte è anche ovunque ed è dove sembra essere assente, in ambienti e comunità in cui il denaro non è una spinta assiologica, che quel capitalismo di piattaforma cercherà le nuove fonti del suo profitto. -

In particolare, la combinazione tra proprietà capitalista dei supporti digitali (ad esempio dei siti di web hosting) e libertà d'uso sfocia in forme di predazione o mercificazione. Nell'aprile 2011 il giornale online Huffington Post è stato acquisito da $A O L$ per 315 milioni di dollari, con la costernazione di migliaia di persone che avevano bloggato gratuitamente e si sono trovate espropriate di centinaia di articoli che avevano pubblicato gratuitamente sul sito del giornale. Diversi milioni di ore di attività dei 9.000 blogger tra il 2005 e il 2011 sono stati speculativamente vaporizzati e sono finiti nelle tasche dei proprietari (Simonet 2018). La cooperazione dei blogger, all'interno di un orizzonte assiologico caratterizzato dall'emulazione, dal riconoscimento e dalla condivisione di uno strumento e di uno spazio per le pubblicazioni, che aveva funzionano come "bene comune" è "sussunto" come ore lavorative non pagate e potenziale valorizzazione economica per la testata giornalistica. $\grave{E}$, cioè, questa attività libera e la sua qualità informativa che ha costituito la forza attrattiva del giornale e lo ha reso un prodotto, ed è questa comunanza cooperativa che è stata la fonte del suo successo tra i lettori. Il "giornale partecipato" che combinava accesso gratuito, numerosi lettori e degli introiti legati quasi esclusivamente alle pubblicità, doveva il suo successo ai blogger che producevano "contenuti di alta qualità a basso costo". Lanciato da Arianna Huffington e Kenneth Lerer con 1 milione di dollari, il giornale ha generato 15 milioni di dollari di entrate pubblicitarie nel 2009 e 65 milioni di dollari nel 2011. Se questi guadagni pubblicitari sono, tra l'altro, il risultato dell'attività dei blogger, sono anche i blogger che hanno determinato in parte l'importo dell'acquisizione (buyout). La rendita legata alla visibilità e al riconoscimento associato a questa qualità dei contenuti gratuiti è stato in definitiva il trampolino speculativo per arricchirsi tramite il supporto digitale. Questa è probabilmente una delle forme più spettacolari di conversione di una cooperazione gratuita in profitto. Ma ogni giorno su YouTube o Twitch, ora la creatività e l'inventiva di massa, ora forme di virtuosismo ludico, si convertono da gratuite a commerciali. Ecco alcuni esempi. Davanti alla telecamera del loro computer, in casa, nella loro camera da letto o sul divano del soggiorno, i giovani disfano il proprio guardaroba, indossano vestiti di marca o si truccano. La durata dell'ascolto del pubblico determina le condizioni per monetizzare il video e, se ha successo, i rappresentanti del marchio supporteranno di più i giovani blogger, fornendo loro altri prodotti, con lo scopo di farseli promuovere (Allard 2019). Con una webcam uno streamer trasmette in diretta la sua partita su un videogioco e la commenta. Riesce così ad avere vari tipi di entrate dalla sua attività di "giocatore": alcuni spettatori pagano per chattare con lui, o anche gli fanno delle donazioni, ma soprattutto riceve una parte delle entrate pubblicitarie di Twitch. Infatti, consuma alcuni prodotti online, come le bevande, e la sua stessa notorietà contribuisce alla reputazione e al successo della piattaforma (Cocq 2018). Come nel caso del blogging e di altre forme di esposizione che possono influire su un progetto professionale o sulla carriera, un'attività in gran parte autotelica associata al riconoscimento - esprimersi, mettersi in mostra, dimostrare il proprio virtuosismo, ecc. - si ibrida con interessi economici e commerciali legati all'attività pubblicitaria e ai guadagni che essa apporta alle piattaforme. Questa ibridazione, in modo surrettizio, devia l'attività in lavoro ma soprattutto si innesta in una zona di informalità per estrarvi profitto. Costituisce una perversione dell'attività vernacolare o di autovalorizzazione in attività di mercato e ci invita a rileggere i molteplici significati dell'informalità. L'informalità non è solo un ambito non regolamentato, è anche lo spazio per un regime di attività che non hanno altro fine che se stesse e le cui ragioni per interagire con gli altri sono in parte estranee all'agire strategico per cui l'altro è un mezzo per un fine. Impadronendosi dell'informale, il capitale converte i fini in mezzi (Marx 1968): assoggettando l'attività autotelica in un mezzo il cui fine esclusivo è fare soldi. La piattaforma come supporto è il trampolino di lancio per questa appropriazione e contemporaneamente per una sottomissione all'accountability. 


\section{LA COLONIZZAZIONE DEL QUOTIDIANO}

Questo primo aspetto dell'informalità del capitale assume un'altra dimensione con le nuove possibilità di ibridare vita quotidiana e mercato, trasformando ancora una volta i fini in mezzi. Le possibilità offerte dalle NICT di drenare dati da tutte le sfere della vita sociale aprono un nuovo campo per la mercificazione. Per esempio, varie piattaforme ("Click and Walk" o BeMyEyes) danno ai loro "collaboratori occasionali" il compito di fornire dati su certi prodotti presentati in negozi o supermercati al fine di raccogliere informazioni utili su di essi per i loro sponsor. Secondo le piattaforme, non si tratta tanto di lavoratori quanto di veri e propri consumatori. Un click walker, dopo aver «scaricato un'applicazione gratuita sul suo smartphone», diventa membro della comunità dei «collaboratori» dell'applicazione e può utilizzare il tempo che trascorre in una strada (street data) o in un supermercato o negozio (store data) per scattare fotografie per verificare la visibilità dei prodotti o la qualità dell'accoglienza, osservare i consumatori e dare un parere. I click walker sono pagati in punti regalo o in euro, mentre i marchi che sponsorizzano i sondaggi, come L'Oréal, Auchan, Décathlon e Nestlé, sono sempre più informati su come i prodotti sono esposti o del livello di gradimento da parte della clientela. Come spiega un'altra piattaforma, BeMyEye, questa attività permette alle persone di «guadagnare soldi nel loro tempo libero». L'espressione da sola è una sintesi della svolta che le piattaforme stanno realizzando. Fino ad ora, la condizione per guadagnare denaro era quella di impegnare il proprio tempo per un periodo di tempo limitato, per poter godere poi del proprio tempo libero. Nell'epoca del capitalismo delle piattaforme, si tratta al contrario di lasciare alle imprese le condizioni per soggiogare i momenti di tempo libero senza contratto per dedicarli a compiti frammentari e banali che permettano di vivere o di guadagnare un po' di soldi. Da una temporalità compatta, circoscritta, inquadrata, il lavoro si vaporizza in una pluralità di momenti più o meno densi, più o meno contenuti. Il rovesciamento non è senza effetti sul rapporto con l'informalità. Non si tratta tanto di attingere a un terreno comune o alla cooperazione come prima, quanto di cogliere una routine quotidiana che può essere ormai una fonte di profitto. Nello stesso modo in cui trasforma una passeggiata in strada o in un supermercato in un compito economicamente significativo, la piattaforma rende informale ciò che prima non lo era. In effetti, per definizione l'informalità presuppone un rapporto: l'informalità ha senso solo in una relazione con il formale; senza non saremmo in grado di distinguere, per esempio, il carattere generalmente deregolamentato del lavoro nel XIX secolo dalle specifiche deregolamentazioni del lavoro nel contesto delle imprese informali in Europa o nelle sweatshops del Bangladesh nel XXI secolo (Sassen 1997). Dal XX secolo la vita quotidiana nei paesi sviluppati non era più oggetto di informalizzazione. Era parte di una sfera autonoma la cui esistenza si basava sulla regolazione e la limitazione dell'orario di lavoro. Infiltrandosi nella vita quotidiana con micro-task, il capitalismo delle piattaforme, sta producendo un'informalità che si espande sempre più in tutte le sfere e agisce come un potente movimento per deregolamentare l'organizzazione temporale delle società sviluppate. Questa colonizzazione del quotidiano estende in modo eclatante quel processo iniziato dalle piattaforme di taxi privato e di car-sharing. Chiunque, in qualsiasi momento, può diventare un micro-tasker di un'attività infinitesimale che lo converte in attore di un'economia non regolata. Il fatto che, in una decisione del 10 febbraio 2020, la Corte d'appello di Douai (Francia) abbia condannato la società Clic and Walk a una multa di 50.000 euro per «lavoro occulto» dimostra la vigilanza dei giuristi riguardo all'indebolimento del significato di lavoro e sembra fare eco alla decisione dello Stato della California secondo cui Uber e Lyft dovrebbero riclassificare i loro autisti come dipendenti ${ }^{4}$. Ma non siamo ancora certamente alla fine di un processo di regolazione la cui posta in gioco è forse più complessa di quanto non appaia. Bisogna chiedersi fino a che punto, in effetti, una riqualificazione del concetto di lavoro potrebbe paradossalmente significare acconsentire alla dinamica di mercificazione della nostra vita quotidiana e fino a che punto. Non dovremmo arrivare a proibire l'intrusione della tecnologia digitale in tutte le sue forme, dai click work al data mining, se vogliamo mantenere uno spazio di vita fuori dal mercato? Se in alcuni contesti di relazione con le piattaforme, per esempio nell'home sharing, l'azione collettiva e l'azione municipale hanno avuto un ruolo decisivo nei processi di regolarizzazione degli affitti e di conseguenza nella direzione di una regolazione dello stesso quotidiano urbano (Aguilera et al. 2019), la situazione è diversa nel contesto del lavoro.

\footnotetext{
${ }^{4} \mathrm{Si}$ sa che questa decisione è stata poi sospesa nello stesso Stato. Vedi sopra.
} 
Per certi versi, l'organizzazione e la messa in comune di esperienze di fronte a clienti senza scrupoli (Irani 2013), la sindacalizzazione (Dufresne 2021), l'azione collettiva e le mobilitazioni (Cant 2019) hanno già avuto luogo, ma si confrontano ancora con le politiche e i governi neoliberali; d'altra parte, è il dare un senso e il misurare le conseguenze del saccheggio dell'intimità domestica che appare come un problema complessivo per lavoratori, cittadini e governanti (Zuboff 2020).

\section{GIOCHI E GESTIONE INFORMALE}

Se il gioco ci è già apparso come poter essere oggetto di profitto pubblicitario attraverso la promozione di articoli (Twitch), ci sembra anche come la molla di una creatività e di un'inventiva immanente alla propria attività, i cui risultati e le scoperte possono essere condivisi tra un'azienda e i giocatori o più semplicemente catturati dall'azienda. L'azienda danese Lego, che dietro i suoi famosi mattoncini apparentemente innocui nasconde una potente azienda, ha creato vari spazi di interattività e scambio con i suoi giocatori che non sono solo bambini. L'uso dei mattoncini che servono per costruire personaggi, oggetti e persino gioielli riguarda anche gli adulti. Questi adulti, infatti, sono mobilitati in un processo cooperativo attorno a un uso ludico dei mattoncini dove diventa particolarmente evidente la tensione tra libero e gratuito. Questo è principalmente un mercato di nicchia in quanto l'uso dei mattoncini di plastica può essere diversificato a seconda delle tipologie e delle comunità di utenti e Lego li invita a creare il proprio design. La possibilità di utilizzare liberamente alcuni strumenti dell'azienda diventa contemporaneamente un'occasione per gli utenti per esprimere la propria creatività, ma anche e conseguentemente una forma di crowdsourcing. Una di queste piattaforme raccoglie idee da utenti-consumatori che possono caricare le loro creazioni su una pagina web. Le loro creazioni, i loro progetti vengono valutati da altri utenti e in base al numero di sostenitori possono essere selezionati dall'azienda. Il dispositivo non solo permette di trarre elementi di innovazione dagli utenti, ma anche di massimizzare la probabilità di avere un prodotto che funzioni (Antorini, Muñiz, 2013). In alcuni casi, l'azienda deve riconoscere i diritti di proprietà intellettuale e alcuni kit personalizzati di parti o modelli indicano esplicitamente chi sono gli inventori e i loro diritti. In questo specifico contesto, vediamo che l'azienda trova tra i fan e tra gruppi periferici know-how, competenze, ma anche socialità cooperative che possono alimentare la sua forza innovativa, potendo anche trarre profitto dalle creazioni e dalle aspirazioni ludiche. I migliori prodotti, nati dalla partecipazione dei clienti alla progettazione di modelli di parti, vengono venduti con il suo marchio. Hobbisti e appassionati del gioco contribuiscono così al suo miglioramento, rendendo porosa la zona di interfaccia tra la ricerca del profitto (Lego) e la ricerca autotelica del piacere ludico, del gioco per il gioco. Molto più che per il lavoro gratuito, i marchi possono beneficiare del lavoro gratuito di alcuni dei loro consumatori o dei loro fan, si tratta di estrarre il risultato di un'esperienza o di un virtuosismo ottenendo un incremento di innovazione particolarmente redditizio sul mercato. A differenza del volontario, che mette a disposizione il proprio tempo libero e talvolta le proprie competenze professionali, fornendo all'azienda una sorta di plusvalore assoluto, l'hobbista virtuoso mette a disposizione il proprio know-how, i suoi "trucchi" e le sue qualità innovative che entrano direttamente in gioco nel processo di creazione, questa volta portando un diverso tipo di profitto. Al contrario dei giochi online di Twitch o anche dell'attività di blogger gratuita dell'Huffington Post, dove la coptazione viene dall'esterno e, in varia misura, si impadronisce dell'attività autonoma, qui la relazione capitalista si innesta sul gioco e si ibrida quasi completamente con esso. Considerando predazione e cooperazione due facce della medaglia, siamo meno in un rapporto di predazione e più di cooperazione, ovvero sul suo lato ludico e autotelico su cui si innesta l'azienda in un processo di collaborazione. Gioco e capitale vivono la stessa vita e sono parte l'uno e l'altro, anche se i loro scopi rimangono distinti. Il capitale entra in relazione simbiotica con l'attività sia per sostenere il giocatore amatoriale sia per dargli il massimo godimento per quanto riguarda la funzione ludica del gioco, ma finisce per nutrirsi della sua creatività, in un rapporto che è contemporaneamente di reciprocità e parassitario. Dati i contesti precedentemente discussi, la penetrazione digitale della sfera privata, le forme aperte di estensione del tempo di lavoro, la predazione delle attività gratuite, la monetizzazione della messa in scena del sé e del desiderio

di riconoscimento, le forme aperte di mobilitazione delle attività quotidiane, in questo caso il rapporto di merci- 
ficazione negozia direttamente con l'attività creativa, si inserisce nella dinamica autotelica del gioco per sostenerla, amplificarla al fine di trarne profitto. La relazione è tale che Lego fa appello ad un management specifico per mobilitare l'attività di hobbisti e dilettanti i cui scopi ovviamente sono spesso diversi da quelli dell'azienda. "Qualunque sia l'approccio scelto da un'azienda, ci sono problemi di collaborazione, come trovare i migliori utenti innovativi e integrare le loro innovazioni. Le comunità di utenti hanno in mente i propri interessi. Essere un hobbista o un fan è qualcosa che ha le sue radici lontane dal mercato. Le loro motivazioni sono diverse da quelle di un'attività a scopo di lucro" (Antorini, Muñiz, 2013). Un management di dilettanti! Bisogna prendere la misura dell'ossimoro contenuto in questa idea, ben in continuità con quanto osservato in precedenza. L'offuscamento di cosa significhi lavoro o ancor più fortemente di cosa esso significhi come esperienza ha la sua estensione in una gestione di utenti hobbisti, di fan con i loro fini specifici, con lo scopo di mobilitarli. Questa gestione dell'informale del nuovo capitalismo potrebbe essere il punto avanzato di un processo che già caratterizza altre sfere del gratuito.

\section{LA FINE CAPITALISTA DEL LAVORO OVVERO L'INFORMALITÀ DEL CAPITALE}

Qualche anno fa si volevano riprendere le teorie dell'audience commodity e sostenere che gli utenti di Internet, proprio come gli utenti della televisione di ieri, lavoravano o creavano valore e plus-valore (Fuchs, 2014). Non c'era bisogno di andare così lontano. Piuttosto che trasformare un'attività passiva, evanescente in un lavoro eventualmente retribuito, è bastato rivolgersi nella direzione opposta al modo in cui il lavoro si è diffuso come attività eteronoma, separata, "eufemizzata" in ambiti che non erano mai stati i suoi luoghi di produzione fino a quel momento. In diversi modi, il capitale ha diluito il lavoro nel tempo sociale dedicato al divertimento (tempo libero) e alle attività quotidiane, per trarre profitto da quei piccoli compiti che hanno perso la consistenza eteronomica di quanto si faceva ieri nelle fabbriche o negli uffici. Qual è la posta in gioco in questo fenomeno? Lo è forse meno nelle condizioni di retribuzione che nelle condizioni di regolazione. Acconsentendo troppo ad allargare il concetto di lavoro alle tracce lasciate dalla nostra navigazione sul web e possibilmente chiedendo di essere pagati per il nostro pubblico, rischiamo di perdere il tema fondamentale del capitalismo di piattaforma: l'alienazione dal quotidiano e la predazione dell'intimo (Harcourt 2020). Ma se le piattaforme sono progressivamente arrivate ad estrarre dati e quindi informazioni dalle tracce che noi produciamo, virtuali o reali che siano, penetrando nella sfera dell'intimo per influenzarla sempre più, la questione sollevata dall' informalità del capitale è distinta. Si tratta anzitutto di una questione storica del capitalismo fin dalla sua nascita: quella del tempo, come condizione di estrazione. Se proprio finora il conflitto sul tempo, come risultato della lotta tra capitale e lavoro, è stato regolato sulla durata dell'orario di lavoro e sui limiti legali e morfologici imposti al lavoro, il capitalismo delle piattaforme appartiene a una dinamica storica di sovvertimento di questi confini per riconfigurare le condizioni di estrazione del profitto. È un cavallo di Troia nelle regolazioni storiche del XX secolo. Come mostrano la maggior parte delle piattaforme di lavoro, in nome della sua intermediazione, da un lato viene negata la subordinazione e il rapporto salariale a beneficio di questa libertà di mercato di cui Marx ha detto da qualche parte è “la libertà di farsi sfruttare”, e dall'altro si invadono costantemente le regolazioni giuridiche, comunitarie e aziendali per farle esplodere. La capacità delle NITC di coordinare pratiche, usi che le tradizionali organizzazioni burocratiche e gerarchiche non possono gestire proficuamente, offre possibilità di profitto a partire da questi compiti infinitesimali, casuali, in ambiti rimasti lontani dal mercato. Proporre dei compiti frammentati per pochi minuti a qualsiasi ora del giorno o della notte, far lavorare dei passanti qualunque a valutare degli articoli esposti sugli scaffali; seguire e razionalizzare le consegne dei fattorini o le corse degli autisti senza doverli organizzare, in uno spazio potenzialmente illimitato, tutti questi compiti sarebbero stati irrealizzabili o incontrollabili fino all'avvento del digitale. È questa possibilità di controllare il caos che è al centro del nuovo rapporto tra piattaforme e informale. Qualsiasi spazio, qualsiasi territorio, qualsiasi sfera sociale è potenzialmente controllabile a distanza; e diventa allora possibile che esse sconfinino l'una nell'altra, in quanto sono finora rimaste senza necessità di regolamentazione, essendo estranee al lavoro stesso. Finché non blocchiamo l'artificio e l'ideologia delle piattaforme, fino a quando non chiameremo queste attività e micro-task come lavoro, con le conseguenze legali ad esso associate, saremo esposti agli effetti deleteri delle piattaforme. Que- 
ste stanno già causando un peggioramento delle condizioni di vita dei lavoratori poco qualificati e di schiere di gig worker, espandendo la figura del lavoratore indipendente. Alla fine, raggiungeranno i professionisti e mineranno la loro capacità di resistere al declino della loro remunerazione dovuto alla concorrenza internazionale o alla competizione con lavoratori amatoriali. Fino a quando non avremo preso una decisione sulla legittimità o illegittimità di questi compiti intrusivi, cioè finché non avremo deciso socialmente sull'utilità sociale di questo tipo di lavoro, in breve, finché non lo avremo regolato per porre fine a questo potere trasgressivo dei confini, l'informalità messa in moto dal capitalismo delle piattaforme non smetterà di crescere e il nostro disagio con essa.

\section{RIFERIMENTI BIBLIOGRAFICI}

Aguilera T., Artioli F., Colomb C. (2019), Les villes contre Airbnb ? Locations meublées de courte durée, plateformes numériques et gouvernance urbaine, in A. Courmont e P. Le Galès, Gouverner la ville numérique, PUF.

Allard L. (2019), Youtubeuse, Youtubeur, travailler à être soi-même à l'âge du talent numérique?, in «Études digitales», 7.

Anderson C. (2012), La longue traine, Paris : Champ Flammarion.

Antorini Yun Mi, Muñiz A. M. (2013), The Benefits and Challenges of Collaborating with User Communities, in «Research-Technology Management», 56, 3: 21-28.

Arcidiacono D., Gandini A., Pais I. (2018), Sharing what? The 'sharing economy' in the sociological debate, in «The Sociological Review Monographs», 66, 2: 275-288.

Barraud de Lagerie P., Sigalo Santos L. (2018), Et pour quelques euros de plus - Le crowdsourcing de micro-tâches et la marchandisation du temps, in «Réseaux», 212.

Cant C. (2019), Riding for Deliveroo. Resistance in the New Economy, Cambridge: Polity.

Casilli A. (2019), En attendant les robots, Paris: Seuil.

Certeau M., de (1980), L'invention du quotidien, Paris: Union générale d'éditions.

Cingolani P. (2016) Ubérisation, turc mécanique, économie à la demande : où va le capitalisme de plateforme?, in «The conversation», 26 août.

Cingolani P. (2017), La précarité, Que-sais-je ? PUF.

Cingolani P. (2021), La colonisation du quotidien, Amsterdam.

Cocq M. (2018), Constitution et exploitation du capital communautaire - Le travail des streamers sur la plateforme Twitch, in «La nouvelle revue du travail», 13.

Davis G. (2016), The Vanishing American Corporation, Oakland: Berrett-Kohler Publishers.

Debord G. (1961), Perspectives de modifications conscientes dans la vie quotidienne, in «Internationale situationniste $\gg, 6$.

Dufresne A., Leterme C. (2021), Travailleurs de plateforme. La lutte pour les droits dans l'économie numérique, Bruxelles : GRESEA.

Fuchs C. (2014), Digital labor and Karl Marx, Routledge.

Harcourt B. (2020), La société d'exposition - désir et désobéissance à l'ère du numérique, Paris : Seuil.

Jounin N. (2008), Chantier interdit au public - enquête parmi les travailleurs du bâtiment, Paris: La découverte.

Illich I. (1981), Le travail fantôme, Paris: Seuil.

Ilo (2021), 2021 - World Employment and Social Outlook - The role of digital labour platforms in transforming the world of work.

Irani L., Six Silberman M. (2013), Turkopticon: Interrupting Worker Invisibility in Amazon Mechanical Turk, in «CHI 2013: Changing Perspectives», Paris.

Kesselman D. (2020), Ballot Issues : la démocratie directe et la société américaine dans les urnes, in «IdeAs», 6.

Marx K. (1968), Manuscrit de 1844, in CEuvres, Économie II, Paris: Gallimard.

O’Connor S. (2013), Amazon Unpacked, in «Financial Times», 8 février.

Paquot T. (2012), Introduction à Ivan Illich, Paris: La Découverte. 
Proudhon P.-J. (1839), De l'utilité de la célébration du dimanche, Besançon: Bintot

Ross A. (2009), Nice work if you can get it: life and labor in precarious times, New York: University Press.

Rosenblat A. (2018), Uberland: how algorithms are rewriting the rules of work, University of California Press.

Sassen, S. (1997), Informalization in advanced market economies, Development Policies Department, International Labour Office.

Simonet M. (2018), Travail gratuit : la nouvelle exploitation ?, Paris: Textuel.

Shirky C. (2009), Here Comes Everybody. The Power of Organizing Without Organizations, New York: Penguin Books.

Weber F. (1985), Le travail à côté, Paris : EHESS.

Weil D. (2014), Fissured Workplace. Why Work Became So Bad for So Many and What Can Be Done, Cambridge: Harvard University Press.

Zuboff S. (2020), L'Áge du capitalisme de surveillance, Zulma. 